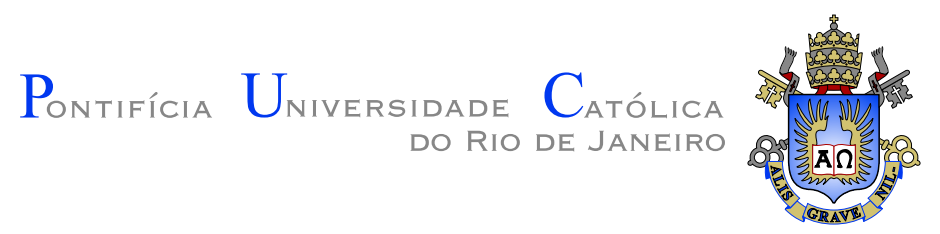

Eduardo Pasquetti

\title{
Métodos Aproximados de Solução de Sistemas
} Dinâmicos Não-Lineares

Tese apresentada como requisito parcial para obtenção do título de Doutor em Engenharia Civil pelo Programa de Pós-graduação em Engenharia Civil da PUC-Rio.

Orientador: Prof. Paulo Batista Gonçalves 


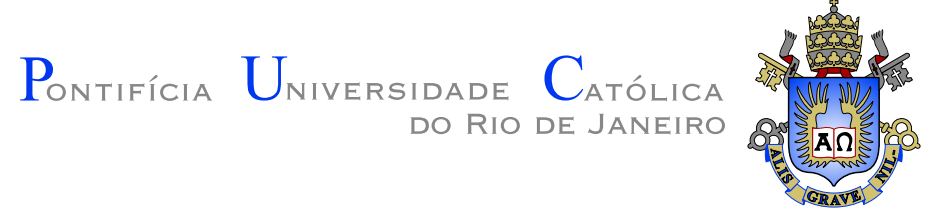

Eduardo Pasquetti

\title{
Métodos Aproximados de Solução de Sistemas Dinâmicos Não-Lineares
}

Tese apresentada como requisito parcial para obtenção do título de Doutor em Engenharia Civil pelo Programa de Pós-graduação em Engenharia Civil da PUC-Rio. Aprovada pela Comissão Examinadora abaixo assinada.

\author{
Prof. Paulo Batista Gonçalves \\ Orientador \\ Pontifícia Universidade Católica do Rio de Janeiro
}

Prof. Djenane Cordeiro Pamplona

PUC-Rio

Prof. José Manoel Balthazar

UNESP

Prof. Marcelo Amorim Savi

COPPE-UFRJ

Prof. Raul Rosas e Silva

PUC-Rio

Prof. José Eugenio Leal

Coordenador Setorial do Centro Técnico Científico - PUC-Rio 
Todos os direitos reservados. É proibida a reprodução total ou parcial do trabalho sem autorização da universidade, do autor e do orientador.

\section{Eduardo Pasquetti}

Graduou-se em Engenharia Civil pela Universidade de Passo Fundo. Obteve o título de Mestre em Estruturas pela Pontifícia Universidade Católica do Rio de Janeiro.

Ficha Catalográfica

Eduardo Pasquetti

Métodos Aproximados de Solução de Sistemas Dinâmicos Não-Lineares/ Eduardo Pasquetti; orientador: Paulo Batista Gonçalves. - 2008.

255 f: il. ; $30 \mathrm{~cm}$

Tese (Doutorado em Engenharia Civil) - Pontifícia Universidade Católica do Rio de Janeiro, Rio de Janeiro, 2008.

Inclui bibliografia.

1. Engenharia Civil - Teses. 2. Dinâmica nãolinear. 3. Métodos aproximados. 4. Não-linearidades não polinomiais. 5. Métodos de perturbação. I. Gonçalves, Paulo Batista. II. Pontifícia Universidade Católica do Rio de Janeiro. Departamento de Engenharia Civil. III. Título. 


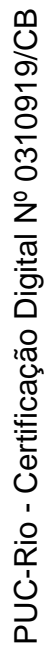

Aos meus pais Luiz e Realda, ao meu irmão Luiz Ricardo e à minha filha Maria Eduarda. 


\section{Agradecimentos}

Ao meu orientador, Paulo Batista Gonçalves, pelo conhecimento transmitido, incentivo, paciência e dedicação ao longo destes sete anos.

À PUC-Rio e aos professores do departamento, por terem me admitido no programa de pós-graduação.

À banca examinadora.

À CNPQ, pelo apoio financeiro.

Aos meus colegas do Tecgraf: Lula, por sua compreensão e paciência ao longo destes últimos anos. À Antônio Sergio, Anderson e Júlio César, pelas dicas de LaTeX.

Pela amizade e agradável convivência, aos amigos de longa data Müller e Ramires, e aos que que ganhei aqui no Rio, em especial a Walter, Sidiclei, Gilmar, Harry, Del Savio, José Roberto, Diego, Frederico, Magnus e Hilton.

Aos professores Agenor, Ignacio, Zacarias e Moacir que lá no início da graduação me incentivaram para que eu chegasse até aqui.

Aos meus tios Darcia e Gerônimo, Adriana e Alceu, obrigado por tudo.

À minha Natália, obrigado por tudo.

À minha família pelo apoio e incentivo. 


\section{Resumo}

Pasquetti, Eduardo; Gonçalves, Paulo Batista. Métodos Aproximados de Solução de Sistemas Dinâmicos Não-Lineares Rio de Janeiro, 2008. 255p. Tese de Doutorado - Departamento de Engenharia Civil, Pontifícia Universidade Católica do Rio de Janeiro.

Sistemas dinâmicos não-lineares são comuns em engenharia. Este tipo de problema é resolvido por integração numérica das equações de movimento ou por métodos analíticos aproximados (métodos de perturbação) ou semianalíticos como o método do Balanço Harmônico. A integração numérica é um processo lento e oneroso em análises paramétricas. Já os outros métodos aproximados são extremamente rápidos, mas são menos precisos e em problemas com certos tipos de não-linearidade, tais como expoentes fracionários, são de difícil, ou impossível, aplicação. Neste trabalho, são apresentados dois métodos alternativos, baseados nas séries de Taylor, para a análise de sistemas não-lineares. No primeiro método, a resposta é escrita em série de Taylor e propriedades de simetria do sistema no espaço de fase são utilizadas para se determinar a relação freqüência-amplitude ou pontos fixos da resposta. No segundo método a solução é escrita em série de Fourier e as amplitudes dos harmônicos são determinadas da mesma forma que os coeficientes da série de Taylor. A simetria do sistema agora fica implícita na solução em série de Fourier, e a relação freqüência-amplitude ou os pontos fixos da resposta são obtidos utilizando equações suplementares. Através de comparações com outros métodos, mostra-se que os métodos desenvolvidos são de fácil implementação e precisos. Estes possuem as vantagens de serem aplicados a problemas com diversos tipos de não-linearidade e de fornecerem uma resposta em série de Fourier onde as amplitudes são determinadas analiticamente resolvendo-se um sistema de equações algébricas lineares.

\section{Palavras-chave}

dinâmica não-linear; métodos aproximados; não-linearidades não polinomiais; métodos de perturbação 


\section{Abstract}

Pasquetti, Eduardo; Gonçalves, Paulo Batista (Advisor). Approximate Solution Methods for Nonlinear Dynamical Systems Rio de Janeiro, 2008.255p. PhD. Thesis - Department of Civil Engineering, Pontifícia Universidade Católica do Rio de Janeiro.

Nonlinear dynamical systems are rather common in engineering. This class of problems is usually solved by numerical integration or through the use of approximate analytical methods (perturbation methods) or semi-analytical methods such as the harmonic balance method. The numerical integration is a slow and cumbersome process in parametric analyses. The other methods are usually extremely fast but they are less precise and their application to problems involving certain types of non-linearity, such as fractional power non-linearities, are difficult or even impossible. In this work two alternative methodologies for the analysis of non-linear dynamical systems, based on Taylor series expansions, are proposed. In the first method, the solution of the initial value problem is obtained by expanding the response in Taylor series and the symmetries of the response in phase space are used to obtain the frequency-amplitude relation or the fixed points of the steady-state response. In the second method the response is written as a Fourier series and the modal amplitudes are obtained using the same methodology used in the previous method for the determination of the coefficients of the Taylor expansion. The symmetries of the response are implicit in the Fourier series, and supplementary equations are proposed for the determination of the frequency-amplitude relation and the fixed points of the response. Comparisons with other existing methods show that the two proposed methods are precise and can be easily applied to the analysis of several dynamical systems. The main advantages of the proposed methods are that they can be applied to several types of non-linearities and that analytic expression for the Fourier coefficients can be obtained by the solution of a system of linear algebraic equations.

\section{Keywords}

nonlinear dynamics; approximate methods; non-polynomial nonlinearities; perturbation methods 


\section{Sumário}

$\begin{array}{lll}\mathbf{1} & \text { Introdução } & \mathbf{2 0}\end{array}$

1.1 Conceitos Básicos em Oscilações Não-Lineares . . . . . . . . . . 25

1.2 Objetivos $\ldots \ldots \ldots \ldots \ldots \ldots \ldots \ldots$

1.3 Novos Métodos Propostos . . . . . . . . . . . . . 30

1.4 Histórico . . . . . . . . . . . . . . . . . . . . . . . . . . . . . . . . . . . . . . . . 31

1.5 Descrição da tese . . . . . . . . . . . . . . . 38

\begin{tabular}{|lll}
2 & Vibração em Sistemas Contínuos & 41
\end{tabular}

2.1 Relação deformação-deslocamentos . . . . . . . . . . . . . . . 41

2.2 Viga contínua - solução linear . . . . . . . . . . . . . . . . . . . . 4 43

2.3 Viga contínua - formulação não-linear . . . . . . . . . . . . . . . . . . 47

2.4 Viga contínua com imperfeição inicial . . . . . . . . . . . . . . 50

2.5 Viga contínua - solução com não-linearidades mais completa\$ . . 52

3 Métodos de Perturbação 54

3.1 Método Lindstedt-Poincaré. . . . . . . . . . . . . . . . 55

3.1 .1 Vibração Forçada . . . . . . . . . . . . . . . . . . . 59

3.1 .2 Vibração Forçada Amortecida . . . . . . . . . . . . . . 62

3.1.3 Programa em Álgebra simbólica . . . . . . . . . . . . . . 64

3.2 Método Lindstedt-Poincaré Modificadd . . . . . . . . . . . . 65

3.2 .1 Vibração Forçada . . . . . . . . . . . . . . . 71

3.2 .2 Vibração Forçada Amortecida . . . . . . . . . . . . . . 73

3.3 Método Múltiplas Escalas . . . . . . . . . . . . . . . . 76

3.3 .1 Vibração Forçada . . . . . . . . . . . . . . . . . . . . . . 81

3.3.2 Programa em Álgebra simbólica . . . . . . . . . . . . . . . 86

3.3 .3 Vibração Forçada Amortecida . . . . . . . . . . . . . . . 87

3.4 Não-Linearidade Quadrática . . . . . . . . . . . . . . . . 91

3.4 .1 Método Lindstedt-Poincaré . . . . . . . . . . . . . . 91

3.4 .2 Método de Lindstedt-Poincaré Modificadd . . . . . . . . 94

$3.4 .3 \quad$ Método das Múltiplas escalas . . . . . . . . . . . . . . 97

4 Método do Balanço Harmônico 101

4.1 Newton-Raphson com Comprimento de Arcd . . . . . . . . . . 102

4.2 Vibração livre . . . . . . . . . . . . . . . . . . . . . . . . 102

4.3 Vibração Forçada . . . . . . . . . . . . . . . . . . . 105

4.3 .1 Solução de sistemas algébricos não-lineares através de método de perturbação . . . . . . . . . . 107

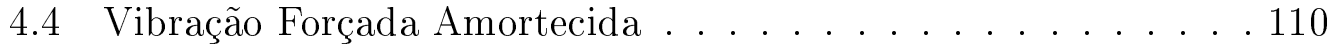

4.5 Método de Galerkin-Urabe . . . . . . . . . . . . . . . . . . . . 111

4.6 Método do balanço harmônico incremental . . . . . . . . . . . . . . . . . . 112

4.7 Não Linearidade Quadrática . . . . . . . . . . . . . . . . . . 114

5 Sistemas Lineares com Coeficientes Periódicos 116 5.1 Multiplicadores de Floquet . . . . . . . . . . . . . . . 117 
5.2 Estabilidade de sistemas não-lineares . . . . . . . . . . . . . . 119

5.2 .1 Determinante de Hill . . . . . . . . . . . . . . . . 120

6 Solução de Sistemas Dinâmicos Não-Lineares por Séries 122

6.1 Séries de Potências . . . . . . . . . . . . . . . . 122

6.2 Método baseado em séries de Taylor. . . . . . . . . . . . . . . . . . . . . . . . . . . . . . . . . .

6.2 .1 Vibração livre . . . . . . . . . . . . . . . . . . . . . . . . 125

6.2 .2 Equação de Duffing . . . . . . . . . . . . . . . . . . 126

6.2 .3 Relação entre os métodos de Taylor e LP . . . . . . . . . 134

6.2.4 Soluções em série de Fourier a partir da série de Taylor . 141

6.2 .5 Aproximações de Padê . . . . . . . . . . . . . 146

6.2 .6 Vibração Forçada . . . . . . . . . . . . . . . . . . . . . . . . . . . . . . . . . . . . . . .

6.2 .7 Equação de Duffing . . . . . . . . . . . . . . . . . . . . . . . . . . . . . . . . . . . . . .

6.2 .8 Validação da Solução em série . . . . . . . . . . . . . . . . . . . . . . . . . . . . . .

6.3 Método de Fourier-Taylor (FT) . . . . . . . . . . . . . . 162

6.3.1 Resolução de problemas de vibração livre. . . . . . . . . . . . . . . . . . . . . . . . . .

6.3 .2 Equação de Duffing . . . . . . . . . . . . . . . . . . 164

6.3.3 Método Fourier-Taylor-Galerkin (FTG) . . . . . . . . . . 167

6.3.4 Resolução de problemas de vibração forçada sem amor-

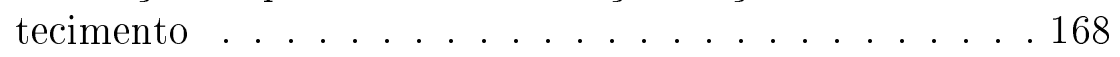

6.3.5 Resolução de problemas de vibração forçada com amor-

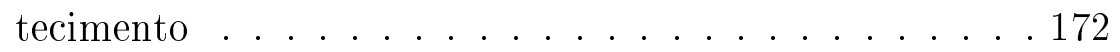

\begin{tabular}{llr}
\hline 7 & Não-linearidades não-polinomiais & 177
\end{tabular}

7.1 Sistema dinâmico com não-linearidade fracionária . . . . . . . 178

$7.1 .1 \quad \ddot{x}+\operatorname{sgn}(x)=0 \ldots \ldots . \ldots . \ldots . \ldots 183$

7.2 Pêndulo plano . . . . . . . . . . . . . . . . . 186

7.3 Pêndulo Elíptico . . . . . . . . . . . . . . . . . . . . . . 188

7.4 Viga com não-linearidades não-polinomiaił . . . . . . . . . . . . 193

7.5 Arco sujeito a uma carga constante aplicada de forma súbitz . . 194

7.6 $\quad$ Equação de Mathieu não-linear . . . . . . . . . . . . . . 197

\begin{tabular}{lll}
\hline Conclusões & 201
\end{tabular}

\begin{tabular}{lll}
\hline & Referências Bibliográficas & 204
\end{tabular}

A Programa em Maple: Lindsted Poincaré modificado - vibração forçada 212

A.1 Rotinas do método da perturbação . . . . . . . . . . . . . . . . . . . . . . . 212

A.2 Equação de Duffing . . . . . . . . . . . . . . . . . . . . 214

A.2.1 Resolve as equações . . . . . . . . . . . . . . . . . . . 216

A.2.2 Relação freqüência-deslocamento . . . . . . . . . . . . . 219

A.2.3 Soluções . . . . . . . . . . . . . . . . . . . . . . . 219

A.2.4 Curva de ressonância . . . . . . . . . . . . . . . . . 220

\begin{tabular}{|lll}
\hline B Programa em Maple: Método de Taylor - vibração livre 223 & 223
\end{tabular}

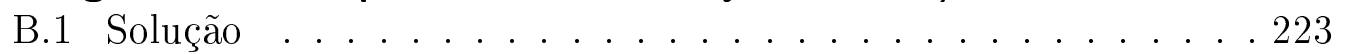

B.2 Relação freqüência-amplitude . . . . . . . . . . . . . . . . . . . . . . . . . . . . . . . . . . . . .

B.3 $\quad$ Exemplo . . . . . . . . . . . . . . . . . . . . . . . 224 
B.3.1 Verificação da solução: integração numérica. . . . . . . . . 225

B.3.2 Transformação da solução em série de Taylor em uma série de Fourier . . . . . . . . . . . . . 226

B.4 Transformação da solução em série de Taylor em uma solução

de Lindstedt-Poincaré . . . . . . . . . . . . . . . . . 229

B.4.1 Determinação das constantes . . . . . . . . . . . 232

C Programa em Maple: Fourier-Taylor - vibração forçada amorte-

cida 234

C.1 Solução . . . . . . . . . . . . . . . . . . . . 234

C.1.1 Transforma a solução em série de Taylor em uma série

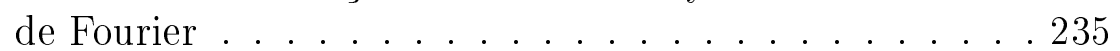

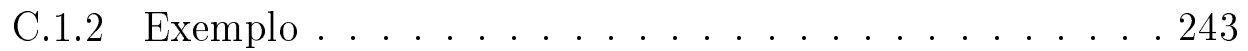

C.2 Exportação de arquivo para programa em C + H . . . . . . . . 246

C.2.1 Escreve o arquivo . . . . . . . . . . . . . . 247

C.2.2 Arquivo exportado . . . . . . . . . . . . . . 250 


\section{Lista de Figuras}

1.1 Relação freqüência-deslocamento inicial, eq. (1-1), sendo $\omega_{0}=1$ e $F(t)=0 . \square, \beta=0 ;+, \beta>0 ; \diamond, \beta<0 \ldots \ldots 26$

1.2 Oscilação livre não-amortecida, eq. (1-1), $\omega_{0}=1$ e $F(t)=0 . \square$, $x_{0}=1$ e $\beta=0 ;+, x_{0}=1$ e $\beta=1 ; \diamond, x_{0}=1,5$ e $\beta=1 . \ldots 26$

1.3 Curvas de ressonância para diferentes valores de não-linearidade do problema (1-1). ................. 27

1.4 Respostas caóticas. Condições iniciais: $\square,(1 ; 0) ;+,(0,99999 ; 0)$. (a) Instantes iniciais; (b) Intervalo de tempo mais distante $\mathrm{do}$ instante inicial. . . . . . . . . . . . . . . . 28

$1.5 \quad \ddot{x}+x+x^{3}=3+F_{1} \cos t$ : (a) Diagrama de Bifurcação; (b) Amplitude máxima. . . . . . . . . . . . . . 29

1.6 Diagrama de Bifurcação em 3d. . . . . . . . . . . . . . . . . . 29

2.1 Deformação de um segmento infinitesimal. . . . . . . . . . . . . 42

2.2 Viga plana sujeita a um carregamento transversal $q(x)$ e axial $P 43$

2.3 Não-linearidade da viga-coluna versus nível de carregamento, . . 49

3.1 Relação $\omega-x_{0} . \square$, RK; +, eq. (3-29); $\diamond$, eq. (3-30); $\triangle$, eq. (3-31). (a) Série convergente, $\omega_{0}=\beta=1$; (b) Série não convergente,

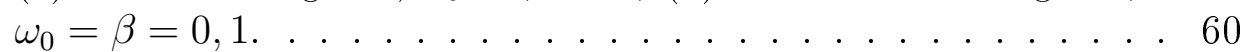

3.2 Relação $\omega-x_{0} . \square$, RK; + , eq. (3-86); $\diamond$, eq. (3-87) . (a) $\omega_{0}=\beta=$

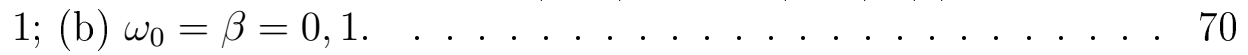

$3.3 \square$, RK; + , aproximação com 2 termos; $\diamond$, aproximação com três termos. (a) versão 1 do método; (b) versão 2. . . . . . . . . . . 86

3.4 Curva de ressonância do problema $\ddot{x}+x+0,1 x^{2}=\cos \Omega t$. $\square$, RK; +, LP com 1 termo; $\diamond$, dois termos; $\triangle$, três termos. . . . . 94

3.5 Deslocamento inicial vs freqüência da resposta. $\square, \mathrm{RK} ;+, \mathrm{LP}$ Modificado com dois termos; $\diamond$, três termos. . . . . . . . . . 96

3.6 LP modificado vs solução numérica do problema $\ddot{x}+x+0,1 x^{2}=$ $\cos \Omega$ t. $\square, \mathrm{RK} ;+, 1$ termo; $\diamond$, dois termos; $\triangle$, três termos. . . . 97

3.7 Deslocamento inicial vs freqüência: $\square$, RK; + , MMS com 2 termos; $\diamond$, três termos. . . . . . . . . . . . . . 100

$4.1 \omega$ vs deslocamento inicial, diversas soluções aproximadas. (a) Uma amplitude é escolhida para ser função das demais amplitudes, $c_{i}=f\left(x_{0}, c_{j}\right)$ para $i \neq j: \square, i=1 ;+, i=2 ; \diamond, i=3 ; \triangle$, $i=4 ; \bigcirc, i=5$. (b) Uma amplitude é escolhida como parâmetro de controle, $c_{i}=$ cte: $\square, i=1 ;+, i=2 ; \diamond, i=3 ; \triangle, i=4 ; \bigcirc$,

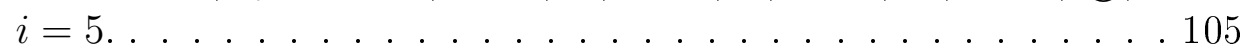

5.1 Espaço de fase . . . . . . . . . . . . . . . . 116

5.2 Relação entre um autovalor de $\Phi$ e um autovalor de $A$. . . . . . 118

5.3 Possibilidades de perda de estabilidade de uma solução periódica 119

6.1 Plano de fase: (a) com dupla simetria; (b) com uma única simetrial 26

6.2 Convergência em $\tau$ das aproximações. $\square$, RK; + , Taylor com cinco termos (eq. (6-33)); $\diamond$, Taylor com seis termos; $\bigcirc$, Taylor com sete termos. . . . . . . . . . . . . . . . 131

6.3 Curva correspondente a um erro inferior ou igual a 0,01\%. . . . 132 
6.4 Soluções aproximadas, $\square$, RK. (a) +, Taylor com cinco termos, eq. (6-35); $\diamond$; LP, solução obtida a partir da aproximação em série de Taylor com cinco termos, eq. 6-60); (b) +, Taylor com seis termos; $\diamond$; LP, solução obtida a partir da aproximação em série de Taylor com seis termos. . . . . . . . . . . . . . 141

6.5 Variação do erro em $\delta$ obtidos com a aproximação em série (dezesseis termos) e com a solução harmônica contruída a partir desta série (quinze harmônicos). . . . . . . . . . . 145

6.6 Solução no tempo do problema $x^{\prime \prime}+x+7,5 x^{3}=0$ para as condições iniciais $(1,0): \square$, RK; + , Taylor com cinco termos; $\diamond$, série de Fourier com quatro harmônicos. . . . . . . . . . . 145

6.7 $\quad$ Plano de fase de um problema forçado amortecido e com nãolinearidade ímpar. . . . . . . . . . . . . . . . 149

6.8 Importância dos termos não-nulos da série de Taylor que representa cos $\Omega t$, avaliados em diferentes instantes de tempo: (a) $\square$, instante $t=0,5 T ; \bigcirc, t=T$; (b) $t=2 T . \ldots . . . . . .162$

6.9 Soluções exata e aproximadas com diferentes números de harmônicos: $\square$, RK; +, FT com dois harmônicos; $\diamond$, FT com três harmônicos; O, FT com quatro harmônicos; . . . . . . . . . . 166

6.10 Resíduo causado por (6-134) para $\omega_{0}=1,5, \beta=3$ e $x_{0}=2$ : $\square$,

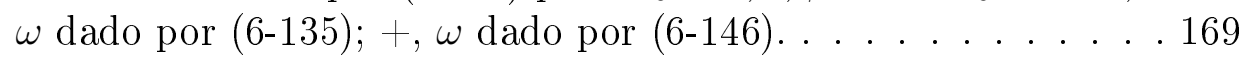

6.11 Resíduo da solução (6-152) para o problema $\ddot{x}+x+x^{3}=$ $50 \cos 5 t: \square, x_{0}$ dado por (6-158);,$+ x_{0}$ obtido ao se conside$\operatorname{rar}(6-159) ; \diamond, x_{0}$ obtido ao se considerar (6-160) . . . . . . . 172

6.12 Curvas de ressonância do problema $\ddot{x}+x+x^{3}=50 \cos \Omega t$ : $\square$, RK; +, HBM com três harmônicos; $\diamond$, FTG com três harmônicos.173

6.13 Variação da coordenada do ponto fixo do problema $\ddot{x}+0,2 \dot{x}+$ $x+3 x^{3}=\cos \Omega t: \square, \mathrm{RK} ;+$, HBM com três pares de harmônicos: $\nabla$, FTG com dois pares de harmônicos. . . . . . . . . . . . 175

6.14 Problema $\ddot{x}+0,4 \dot{x}+x+x^{3}=50 \cos \Omega t$. $\square$, RK; + , HBM com três pares de harmônicos; $\diamond$, FT com 10 pares de harmônicos; (a) Curva $x_{0}-\Omega$; (b) curva $v_{0}-\Omega$. . . . . . . . . . 175

$7.1 \quad$ Simetria no campo de deslocamentos. $\square, q=1 ;+, q<1 ; \diamond q>1.179$

7.2 Energia potencial. $\square, q=0 ;+, q=0,5 ; \diamond, q=1 ; \triangle, q=10$. . . 179

7.3 Curva freqüência-deslocamento inicial. $\square, \mathrm{RK} ;+$, dois termos, eq. (7-5); $\diamond$, quatro termos, eq. (7-7): (a) $\ddot{x}+\operatorname{sgn}(x)|x|^{4 / 3}=0$; (b) $\ddot{x}+\operatorname{sgn}(x)|x|^{3 / 4}=0 \ldots \ldots \ldots$. . . . . . . . . . 182

7.4 Influência da não-linearidade para diferentes deslocamentos iniciais: $\square$, RK e $x_{0}=2 ; \diamond$, FT com sete harmônicos e $x_{0}=2 ;+$, RK e $x_{0}=0,5 ; \triangle$, FT com sete harmônicos e $x_{0}=0,5$. . . . . . 184

7.5 Solução no tempo do problema $\ddot{x}+\operatorname{sgn}(x)=0: \square$, RK; + , eq. (7-11); $\diamond$, eq. (7-17); $\triangle$, eq. (7-18). . . . . . . . 186

7.6 Curva freqüência-deslocamento inicial do problema $\ddot{x}+\sin x=0$ : $\square$, RK; +, eq. (7-22); $\diamond$, eq. (7-21); $\triangle$, eq. (7-23). . . . . . . 188

7.7 Curva freqüência- $g / l: \square$, RK e $\theta_{0}=85,7^{\circ} ;+$, Taylor com 5 termos; $\diamond$, RK $\theta_{0}=5^{\circ} ; \triangle$, FT com cinco harmônicos. . . . . . . 189 
7.8 Respostas no plano de fase do problema $\ddot{x}+\sin x=0$ : (a) $\theta_{0}=86^{\circ}, \square, \mathrm{RK} ;+$, FT com cinco harmônicos; (b) $\theta_{0}=170^{\circ}$, $\square$, RK; + , FT com oito harmônicos; $\diamond$, FT com doze harmônicos.189

7.9 Pêndulo elíptico. . . . . . . . . . . . . . . . . . . . . . . 190

7.10 Curva $\omega_{2}-\theta_{0}:$ (a) $m_{A}=100 m_{B}=1$. $\square$, RK - pêndulo 1gl; + , RK - pêndulo 2gl; $\diamond$, FT com dois harmônicos. (b) $m_{A}=1 m_{B}=1$. $\square$, RK; +, FT com dois harmônicos, eq. (7-39); $\diamond$, Taylor com três termos, eq. (7-35). . . . . . . . . . . . . . 193

7.11 Solução no tempo da eq. (7-40) com os seguintes parâmetros: $x_{0}=2, P=69311,51, E I=5672067, q=0$ e $m=1 . \square, \mathrm{RK}$; + , aproximação com 3 termos; $\diamond, 5$ termos; $\triangle, 7$ termos. . . . . 194

7.12 Curva freqüência-deslocamento inicial do problema (7-40) com os seguintes parâmetros: $P=69311,51, E I=5672067, q=0$ e $m=1 . \square$, RK; +, Taylor com três termos; $\diamond$, Taylor com quatro termos; $\triangle$, FT com três harmônicos. . . . . . . . . . . . . 195

7.13 Sistema idealizado com um grau de liberdade . . . . . . . . . 195

7.14 Respostas no plano fase: (a) $\square, q=0,2 ;+, q=0,42$; (b) $q=0,6196$

7.15 Curva freqüência-carregamento. $\square$, RK; +, eq. (7-46); $\diamond$, Taylor com 5 termos; $\triangle$, eq. (7-48) . . . . . . . . . . . . . . 198

7.16 Espaço de fase do problema $\ddot{\epsilon}+0,2 \dot{\epsilon}+\left(1+9,78519(\cos 2 t)^{2}\right) \epsilon-$ $(5,41808 \cos 2 t) \epsilon^{2}+\epsilon^{3}=0 . \square, \mathrm{RK} ;+, \mathrm{FT}$ com três pares de harmônicos; $\diamond, \mathrm{FT}$ com cinco pares de harmônicos. . . . . . . 200 


\section{Lista de Tabelas}

6.1 Convergência da solução linear. . . . . . . . . . . . . . . . 130

6.2 Convergência da solução não-linear para $b=1$. . . . . . . . . 130

6.3 Convergência da solução não-linear para $b=1,1 . \quad \ldots$. . . . . 131

6.4 Máximos valores do parâmetro de não-linearidade, $b$, para que as aproximações apresentem um erro de aproximadamente 0,01\%

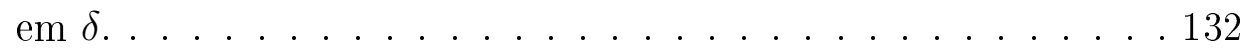

6.5 Diferenças entre as amplitudes dos harmônicos da série de Fourier, obtida a partir da série de Taylor, e da solução obtida com o HBM. . . . . . . . . . . . . . . . . . . . 144

6.6 Diferenças entre as amplitudes dos harmônicos da série de Fourier, obtida a partir da série de Taylor, e da solução obtida pelo HBM. . . . . . . . . . . . . . . . . . . . 144

6.7 Convergência da série de Padé (solução linear). . . . . . . . . . 147

6.8 Convergência da série de Padé do problema não-linear, $b=1$. . . 147

6.9 Convergência da série de Padé, tendo um número variável de termos no numerador, do problema não-linear $b=1$. . . . . . . . 148

6.10 Convergência da relação $f-\delta$ aproximada do problema linear não-amortecido. . . . . . . . . . . . . . 153

6.11 Convergência da solução linear amortecida em $t=T / 2$ do problema $\ddot{x}+0,1 \dot{x}+x=10 \cos 2 t$. . . . . . . . 154

6.12 Convergência das coordenadas do ponto fixo da solução de $\ddot{x}+0,1 \dot{x}+x=10 \cos 2 t$. . . . . . . . . . . 155

6.13 Convergência da solução de $\ddot{x}+0,1 \dot{x}+x=10 \cos (2 t+\phi)$. . . 155

6.14 Convergência do deslocamento máximo e $\phi$ do problema $\ddot{x}+$ $0,1 \dot{x}+x=10 \cos (2 t+\phi) . \ldots \ldots \ldots \ldots . \ldots \ldots 6$

6.15 Convergência do instante $t$ que limita o erro da aproximação a menos de $0,01 \%$ do ponto fixo do problema $\ddot{x}+x+x^{3}=0,5 \cos t .156$

6.16 Convergência do cálculo do ponto fixo do problema $\ddot{x}+x+x^{3}=$

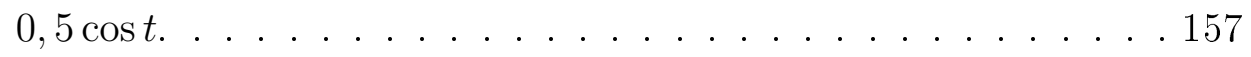

6.17 Convergência da solução do problema $\ddot{x}+x+x^{3}=0,5 \cos t$. .157

6.18 Convergência da solução do problema $\ddot{x}+x+2,5 x^{3}=2 \cos 2 t$. 157

6.19 Convergência da solução do problema $\ddot{x}+x+0,8 x^{3}=10 \cos 2 t$. 157

6.20 Convergência da solução não-linear amortecida, eqs. (6-83) . . . . 158

6.21 Convergência da solução não-linear amortecida, eqs. (6-86) . . . . 158

6.22 Comparação entre as amplitudes da solução obtida com o HBM e da solução em série de Fourier, obtida a partir da solução em série de Taylor. . . . . . . . . . . . . . . . 160

7.1 Resultados obtidos com o método de Taylor para $x_{0}=1 \mathrm{e}$ diferentes não-linearidades e aproximações, comparados com os apresentados por Gottlieb [1]. . . . . . . . . . . . . . 181

7.2 Resultados obtidos com o FT para diferentes aproximações tendo $x_{0}=1$, comparados a solução exata. . . . . . . . . . 183

7.3 Resultados obtidos com o método de Fourier Taylor e o erro em relação a solução exata, eq. (7-13). . . . . . . . . . . 186 


\section{Lista de Símbolos}

\section{Caracteres Romanos}

$A \longrightarrow$ Área, amplitude da resposta, matriz dos coeficientes

$a \longrightarrow$ Amplitude da resposta, amplitude de harmônico

$a_{i} \longrightarrow$ Coordenadas generalizadas, amplitude do harmônico $i$

$\overline{A B} \longrightarrow$ Segmento que une os pontos $A$ e $B$

$\overline{A B}_{x} \longrightarrow$ Componente segundo $x$ do segmento $A B$

$\overline{A B}_{y} \longrightarrow$ Componente segundo $y$ do segmento $A B$

$\overline{A^{\prime} B^{\prime}} \longrightarrow$ Segmento que une os pontos $A^{\prime}$ e $B^{\prime}$

$B \longrightarrow$ Amplitude da resposta

$b \longrightarrow$ Amplitude de harmônico, parâmetro adimensional de nãolinearidade cúbica

$C_{i} \longrightarrow$ Constantes de integração

$c_{i} \longrightarrow$ Amplitude dos harmônico $i$, parcela que compõe a amplitude de um harmônico

$d_{i} \longrightarrow$ Amplitude dos harmônico $i$, parcela que compõe a amplitude de um harmônico

$d x \longrightarrow$ Elemento infinitesimal segundo a direção $x$

$d s \longrightarrow$ Elemento infinitesimal $d x$ após sofrer deformação e deslocamentos

$d_{x} \longrightarrow$ Deslocamento segundo $x$

$d_{y} \longrightarrow$ Deslocamento segundo $y$

$d_{x}^{A} \longrightarrow$ Deslocamento segundo $x$ do ponto $A$ 
$d_{y}^{A} \longrightarrow$ Deslocamento segundo $y$ do ponto $A$

$d_{x}^{B} \longrightarrow$ Deslocamento segundo $x$ do ponto $B$

$d_{y}^{B} \longrightarrow$ Deslocamento segundo $y$ do ponto $B$

$d U \longrightarrow$ Energia interna de deformação de um elemento infinitesimal

$E \quad \longrightarrow$ Módulo de Young do material

$e_{i} \longrightarrow$ Parcela que compõe a freqüência da resposta

$E I \longrightarrow$ Rigidez a flexão

$F \quad \longrightarrow$ Funcional, amplitude da excitação

$f \quad \longrightarrow$ Parâmetro adimensional de força

$F_{0} \longrightarrow$ Parcela constante da excitação

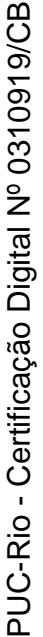

$F_{1} \longrightarrow$ Parcela variável da excitação

$g \longrightarrow$ Constante gravitacional

$I \quad \longrightarrow$ Funcional

$i \quad \longrightarrow$ Componente imaginária

$L \quad \longrightarrow$ Lagrangiano

$l \quad \longrightarrow$ Comprimento da viga, comprimento do pêndulo

$m \longrightarrow$ Massa

$P \longrightarrow$ Carregamento axial, série de Padé

$P(t) \longrightarrow$ Função periódica

$q \longrightarrow$ Carregamento lateral, não-linearidade

$R \longrightarrow$ Resíduo, matriz, raio de convergência

$T \longrightarrow$ Período da resposta

$\bar{T} \longrightarrow$ Energia cinética

$t \quad \longrightarrow$ Tempo

$T_{i} \longrightarrow$ Escalas de tempo 
$t_{0} \longrightarrow$ Instante inicial

$U \longrightarrow$ Energia interna de deformação da estrutura

$u \longrightarrow$ Deslocamento

$u_{0} \longrightarrow$ Imperfeição inicial

$V \quad \longrightarrow$ Potencial

$v \quad \longrightarrow$ Parâmetro adimensional de velocidade

$v_{0} \longrightarrow$ Condição inicial de velocidade

$x, x(t) \longrightarrow$ Deslocamento

$x_{i}(t) \longrightarrow$ Função de deslocamento que compõe a resposta $x(t)$

$\dot{x} \longrightarrow$ Velocidade

$x_{0} \longrightarrow$ Condição inicial de deslocamento

$W, W_{i} \longrightarrow$ Função peso

$w \quad \longrightarrow$ Deslocamento

$w_{i} \longrightarrow$ Parcela que compõe a freqüência da resposta

$w_{0} \longrightarrow$ Imperfeição inicial

$X \longrightarrow$ Matriz de soluções

\section{Caracteres Gregos}

$\alpha \longrightarrow$ Parâmetro de não-linearidade quadrática, numerador da nãolinearidade fracionária

$\beta \longrightarrow$ Parâmetro de não-linearidade cúbica, denominador da nãolinearidade fracionária

$\Delta \longrightarrow$ Deslocamento da carga $P$

$\delta \quad \longrightarrow$ Perturbação, relação entre as freqüências $\left(\omega / \omega_{0}\right)$

$\delta^{1} \longrightarrow$ Primeira variação do funcional

$\delta_{w} \quad \longrightarrow$ Variação de $w$ 
$\delta_{w}^{\prime} \quad \longrightarrow$ Variação de $w^{\prime}$

$\epsilon \quad \longrightarrow$ Deformação específica, perturbação

$\epsilon_{0} \longrightarrow$ Deformação específica da linha neutra, perturbação inicial

$\Phi \quad \longrightarrow$ Matriz de transição

$\phi \quad \longrightarrow$ Ângulo de fase

$\phi(t) \longrightarrow$ Função periódica

$\phi_{i} \longrightarrow$ Modos naturais de vibração

$\phi_{1} \longrightarrow$ Ângulo de fase

$\gamma \quad \longrightarrow$ Ângulo de fase

$\lambda \longrightarrow$ Autovalor

$\varphi \quad \longrightarrow$ Resíduo

$\mu \longrightarrow$ Expoente característico

$\Omega \quad \longrightarrow$ Freqüência da excitação

$\omega \quad \longrightarrow$ Freqüência da resposta

$\omega_{0} \longrightarrow$ Freqüência natural da equação linear

$\Pi \quad \longrightarrow$ Energia potencial total

$\theta \quad \longrightarrow$ Ângulo de rotação

$\theta_{0} \longrightarrow$ Imperfeição inicial, ângulo inicial

$\rho \quad \longrightarrow$ Densidade

$\sigma \quad \longrightarrow$ Parâmetro de sintonia (do Inglês detuning parameter)

$\tau \quad \longrightarrow$ Tempo adimensionalizado

$\zeta \quad \longrightarrow$ Coeficiente de amortecimento 


\title{
Sobrescritos e Subscritos
}

\author{
$\Delta_{()} \longrightarrow$ Variação \\ ()$^{\prime} \longrightarrow$ Derivada em relação a $\mathrm{x}$, derivada em relação a $\tau$ \\ ()$_{\max } \longrightarrow$ Valor máximo \\ ()$^{*} \quad \longrightarrow$ Coordenada de ponto fixo
}

\section{Siglas e Abreviaturas}

$F T \longrightarrow$ Método de Fourier-Taylor

$H B M \longrightarrow$ Método do Balanço Harmônico (do inglês harmonic balance method)

$K B M \longrightarrow$ Método de Krylov-Bogoliubov-Mitropolski

$L P \longrightarrow$ Método de Lindstedt-Poincaré

$M M S \longrightarrow$ Método das Múltiplas Escalas (do inglês method of multiple scales) 